\title{
Studien über $p$-0xydesoxybenzoin
}

\author{
ron
}

\section{Siegmund Weisl.}

Aus dem Laboratorium für chemische Technologie organischer Stoffe an der k. k. Technischen Hochschule in Wien.

(Vorgelegt in der Sitzung am 11. Mai 1905.)

\section{A. Theoretischer Teil.}

Die Synthese aromatischer Oxyketone wurde zuerst von $\mathrm{Nencki}$ und Sieber ${ }^{1}$ versucht, indem sie Eisessig und Phenol oder Eisessig und Resorcin, oder andere mehrwertige Phenole mit Hilfe von Zinkchlorid kondensierten.

So entsteht z. B. das Dioxyacetophenon, auch Resacetophenon genannt, nach der Gleichung:

$$
\mathrm{CH}_{3} \mathrm{COOH}+\mathrm{C}_{6} \mathrm{H}_{4}(\mathrm{OH})_{2}=\mathrm{CH}_{3}-\mathrm{CO}-\mathrm{C}_{6} \mathrm{H}_{3}(\mathrm{OH})_{2}+\mathrm{H}_{2} \mathrm{O} \text {. }
$$

Später hat Rasinsky ${ }^{2}$ darauf hingewiesen, daß derartige Körper aus ihren Komponenten unter Verwendung von Phosphoroxychlorid als Kondensationsmittel entstehen können, daß aber die Reaktion nicht immer im gewünschten Sinne, sondern auch nach der folgenden Gleichung verlaufen könne:

$$
2 \mathrm{CH}_{3} \mathrm{COOH}+\mathrm{C}_{6} \mathrm{H}_{4}(\mathrm{OH})_{2}=\left(\mathrm{CH}_{3} \mathrm{COO}\right)_{2} \mathrm{C}_{6} \mathrm{H}_{4}+2 \mathrm{H}_{2} \mathrm{O} \text {, }
$$

daß also das Säureradikal in die Hydroxylgruppe des Phenols eintritt, unter Bildung von Phenylestern der verwendeten Säure.

Herr Prof. Dr. W. Suida hat mich beauftragt, zu untersuchen, ob die erstgenannte Reaktion auch dann durchführ-

1 Journ. f. prakt. Chemie, (2) 23, 147; (2) 25, 282.

2 Journ. f. prakt. Chemie. (2) 26, 62; (2) 31, 467. 
bar ist, wenn man anstatt der Essigsäure eine substituierte Säure, z. B. die Phenylessigsäure, als eine Komponente verwendet.

Bei Verwendung von Phenol als zweite Komponente würde die Reaktionsgleichung lauten:

$$
\begin{aligned}
\mathrm{C}_{6} \mathrm{H}_{5} \mathrm{CH}_{2}-\mathrm{CO} \mathrm{OH}+\mathrm{H} & \mathrm{C}_{6} \mathrm{H}_{4} \mathrm{OH}= \\
& =\mathrm{C}_{6} \mathrm{H}_{5}-\mathrm{CH}_{2}-\mathrm{CO}-\mathrm{C}_{6} \mathrm{H}_{4} \mathrm{OH}+\mathrm{H}_{2} \mathrm{O} .
\end{aligned}
$$

Man gelangt wieder zu einem Körper, der als Oxyketon aufgefaßt werden kann, der aber zweckmäßiger von der Diphenyläthangruppe abgeleitet wird und dann als Oxydesoxybenzoin aufzufassen wäre, indem im Desoxybenzoin $\mathrm{C}_{6} \mathrm{H}_{5}-\mathrm{CH}_{2}-\mathrm{CO}-\mathrm{C}_{6} \mathrm{H}_{5}$ in jenem Kern, der der Carbonylgruppe benachbart ist, ein Wasserstoffatom durch die Hydroxylgruppe ersetzt erscheint.

Es gelang mir einen derartigen Körper darzustellen, indem ich die theoretischen Mengen von Phenol und Phenylessigsäure zusammenschmolz und in die am Rückflußkühler erhitzte Flüssigkeit die dreifache Gewichtsmenge der Phenylessigsäure an frisch umgeschmolzenem und gepulvertem Zinkchlorid in kleinen Portionen eintrug.

Da ich, auf diese Weise vorgehend, keine befriedigenden Ausbeuten erhielt, weil man zum größten Teile die unveränderten Komponenten aus der Reaktionsmasse zurückerhält, so versuchte ich, andere Kondensationsmittel in Verwendung zu ziehen.

Aber sowohl Phosphoroxychlorid als auch Schwefelsäure, endlich Zinntetrachlorid führten bei zahlreichen ausgeführten Versuchen nicht zum gewünschten Ziele, da immer Verharzung des Reaktionsgemisches eintrat.

Ich gab daher weitere Versuche in dieser Richtung hin auf und versuchte auf einem anderen Wege zum Oxydesoxybenzoin zu gelangen.

Es ist bekannt, daß es Gräbe und Bungen er ${ }^{1}$ gelungen ist, Desoxybenzoin nach der Friedel-Crafts'schen Reaktion aus

1 Berl. Ber., 12, 1080. Vergl. Berl. Ber., 14, 1645; 15, 1680; 22, 1231, 24,$3541 ; 25,2239 ; 32,1564$. 
Phenylessigsäurechlorid, Benzol und Aluminiumchlorid darzustellen. Die Reaktionsgleichung lautet:

$\mathrm{C}_{6} \mathrm{H}_{5}-\mathrm{CH}_{2}-\mathrm{COCl}+\mathrm{C}_{6} \mathrm{H}_{6}=\mathrm{C}_{6} \mathrm{H}_{5}-\mathrm{CH}_{2}-\mathrm{CO}-\mathrm{C}_{6} \mathrm{H}_{5}+\mathrm{HCl}$.

Andrerseits hat Behn, ${ }^{1}$ von dem Gedanken ausgehend, daß Nitrokohlenwasserstoffe ${ }^{2}$ nach der Friedel-Crafts'schen Reaktion gar nicht reagieren, versucht, solche Nitrokohlenwasserstoffe (wie Nitrobenzol) als Verdünnungsmittel bei dieser Reaktion zu verwenden, wobei er auch die Beobachtung machte, daß die bei dieser Synthese entstehenden Aluminiumdoppelverbindungen sehr gut in Nitrobenzol löslich sind.

So hat Behn unter Anwendung von Nitrobenzol als Verdünnungsmittel aus Acetylchlorid, Thymol und Aluminiumchlorid das entsprechende Phenolketon erhalten, wobei die erzielten Ausbeuten nahezu quantitativ genannt werden.

Es lag nun der Gedanke nahe, die Friedel-Crafts'sche Reaktion in der von Behn modifizierten Form für die Synthese des Oxydesoxybenzoins zu verwerten.

Die entsprechende Reaktionsgleichung lautet dann:

$$
\begin{aligned}
\mathrm{C}_{6} \mathrm{H}_{5}-\mathrm{CH}_{2}-\mathrm{COCl}+\mathrm{C}_{6} \mathrm{H}_{5} \mathrm{OH} & = \\
& =\mathrm{C}_{6} \mathrm{H}_{5}-\mathrm{CH}_{2}-\mathrm{CO}-\mathrm{C}_{6} \mathrm{H}_{4} \mathrm{OH}+\mathrm{HCl} .
\end{aligned}
$$

Tatsächlich erhielt ich durch Kondensation von Phenylessigsäurechlorid, Phenol und Aluminiumchlorid in Gegenwart von Nitrobenzol einen Körper, der sich bei näherer Untersuchung als identisch mit dem Oxydesoxybenzoin erwies, welches ich früher aus Phenol und Phenylessigsäure vermittels Zinkchlorid dargestellt hatte.

Die zweite Methode gibt 60 bis 70 prozentige Ausbeuten, während ich nach der ersten Methode nur 20\% der Theorie an reinem Oxydesoxybenzoin erhalten konnte.

Macht schon die Darstellung des $p$-Oxydesoxybenzoins aus Phenylessigsäurechlorid die angenommene Konstitution sehr wahrscheinlich, so wurde selbe noch weiterhin sichergestellt durch den Nachweis der charakteristischen Gruppen.

\footnotetext{
1 D. R. P. 95901.

2 Berl. Ber., 25, 3523.
} 
Der Nachweis der freien phenolischen Hydroxylgruppe wurde durch Acetylierung, der Nachweis der Ketogruppe durch Darstellung des Oxims erbracht. Endlich nahm ich die Spaltung des Oxydesoxybenzoins mit Kalilauge vor und erhielt dabei nach der Gleichung:

$$
\mathrm{C}_{6} \mathrm{H}_{5}-\mathrm{CH}_{2} \frac{\vdots}{\mathrm{CO}}-\mathrm{C}_{6} \mathrm{H}_{4} \mathrm{OH}+\mathrm{H}_{2} \mathrm{O}=\mathrm{C}_{6} \mathrm{H}_{5} \mathrm{CH}_{3}+\mathrm{C}_{6} \mathrm{H}_{4}\langle\mathrm{OOOH}
$$

Toluol und $p$-Oxybenzoesäure.

Die Stellung der Hydroxylgruppe zum Phenylessigsäurereste ist daher die »para «-Stellung. Diese Stellung war auch die theoretisch wahrscheinlichste. Der neue Körper ist daher ein $p$-Oxydesoxybenzoin.

Als Derivat eines Desoxybenzoins waren bei dem gewonnenen Produkte vor allem die Beziehungen interessant, die sich zwischen ihm und seinen Oxydations- und Reduktionsprodukten ergeben würden.

Was zunächst die Oxydation betrifft, so ist bekannt, daß die Stammsubstanz der ganzen Gruppe das Diphenyläthan $\mathrm{C}_{6} \mathrm{H}_{5}-\mathrm{CH}_{2}-\mathrm{CH}_{2}-\mathrm{C}_{6} \mathrm{H}_{5}$ bei der Oxydation in Benzil $\mathrm{C}_{6} \mathrm{H}_{5}-$ $-\mathrm{CO}-\mathrm{CO}-\mathrm{C}_{6} \mathrm{H}_{5}$ übergeht. In gleicher Weise lassen sich das Benzoin $\mathrm{C}_{6} \mathrm{H}_{5}-\mathrm{C}<\mathrm{OH}-\mathrm{CO}-\mathrm{C}_{6} \mathrm{H}_{5}$ und das Hydrobenzoin $\begin{array}{cc}\mathrm{H} & \mathrm{H} \\ \mathrm{C}_{6} \mathrm{H}_{5}-\mathrm{C}-\mathrm{C}-\mathrm{C}-\mathrm{C}_{6} \mathrm{H}_{5} \text { in Benzil überführen. Das Desoxybenzoin } & \mathrm{OH} \mathrm{OH}\end{array}$ verhält sich anders; es zerfällt bei der Oxydation in zwei Moleküle Benzoesäure.

Wie schon List ${ }^{1}$ gezeigt hat, ist aber eine Überführung in Benzil doch möglich, wenn man Mononitrodesoxybenzoin $\mathrm{NO}_{2}-\mathrm{C}_{6} \mathrm{H}_{4}-\mathrm{CH}_{2}-\mathrm{CO}-\mathrm{C}_{6} \mathrm{H}_{5}$ mit Chromsäure und Eisessig oxydiert. Man erhält dann Nitrobenzil

$$
\mathrm{NO}_{2}-\mathrm{C}_{6} \mathrm{H}_{4}-\mathrm{CO}-\mathrm{CO}-\mathrm{C}_{6} \mathrm{H}_{5} \text {. }
$$

Es gelang mir nun auch, das $p$-Oxydesoxybenzoin durch vorsichtig geführte Oxydation in das entsprechende $p$-Oxy-

1 Berl. Ber., 26, 2453. Vergl. Berl. Ber., 21, 1304. 
benzil $\mathrm{C}_{6} \mathrm{H}_{5}-\mathrm{CO}-\mathrm{CO}-\mathrm{C}_{6} \mathrm{H}_{4}-\mathrm{OH}$ überzuführen, wobei als Oxydationsmittel ebenfalls Chromsäure verwendet wurde.

Als $\alpha$-Diketon gibt das $p$-Oxybenzil die charakteristische Farbenreaktion von E. B a mberger ${ }^{1}$ mit Kalilauge.

Die beiden Ketogruppen wies ich durch Darstellung eines $p$-Oxybenzildioxims nach. Theoretisch sind mehrere stereoisomere Dioxime ${ }^{2}$ vorauszusehen.

Bezüglich der Reduktion des $p$-Oxydesoxybenzoins, muß ich folgende, bereits bekannte Tatsachen vorausschicken.

Limpricht und Schwanert ${ }^{3}$ haben zuerst versucht, das Desoxybenzoin zu reduzieren. Faßt man es als Keton auf, so hat man bei der Reduktion die Bildung eines sekundären Alkohols, in diesem Falle das Toluylenhydrat $\mathrm{C}_{6} \mathrm{H}_{5}-\mathrm{CH}_{2}-\mathrm{CH}(\mathrm{OH})-\mathrm{C}_{6} \mathrm{H}_{5}$ zu erwarten.

Den beiden Forschern gelang es durch Reduktion des Desoxybenzoins mit Natriumamalgam den genannten Körper zu erhalten, wobei als Nebenprodukte die beiden isomeren Desoxybenzoinpinakone von der allgemeinen Formel

$$
\begin{aligned}
& \mathrm{C}_{6} \mathrm{H}_{5}-\mathrm{CH}_{2}-\mathrm{C}-(\mathrm{OH})-\mathrm{C}_{6} \mathrm{H}_{5} \\
& \mathrm{CH}_{5}-\mathrm{C}_{6} \mathrm{H}_{2}-\mathrm{C}-(\mathrm{OH})-\mathrm{C}_{6} \mathrm{H}_{5}
\end{aligned}
$$

enstanden.

Zagumeny ${ }^{4}$ und später Blan $\mathrm{k}^{5}$ haben gezeigt, daß die Desoxybenzoinpinakone vornehmlich dann entstehen, wenn die Wasserstoffentwicklung bei der Reduktion langsam verläuft, also besonders bei Verwendung von Zinkstaub und Kalilauge oder Zinkstaub und Eisessig oder Salzsäure.

Beim $p$-Oxydesoxybenzoin war anzunehmen, daß bei der Reduktion ähnliche Verhältnisse obwalten würden. Bei der Reduktion mit Natriumamalgam erhielt ich aber weder den entsprechenden sekundären Alkohol noch ein Oxypinakon, sondern es entstand eine Substanz, die einen größeren Kohlen-

1 Berl. Ber., 18,865 .

2 Berl. Ber., 16, 503, 2176; 21, 784, 1303, 3510; 25, 2169.

3 Liebig's Annalen, 155, 62.

4 Berl. Ber., 5, 1102; 7, 1651 .

5. Lie big's Annalen, $248,6$. 
stoffgehalt als das erwartete Oxytoluylenhydrat $\mathrm{C}_{6} \mathrm{H}_{5}-\mathrm{CH}_{2}-$ $-\mathrm{CH}(\mathrm{OH})-\mathrm{C}_{6} \mathrm{H}_{4} \mathrm{OH}$ und auch einen anderen Wasserstoffgehalt aufwies und der die empirische Formel $\mathrm{C}_{29} \mathrm{H}_{27} \mathrm{O}_{3}$ zuzuschreiben ist, während dem Oxytoluylenhydrat die Formel $\mathrm{C}_{14} \mathrm{H}_{14} \mathrm{O}_{2}$ und dem Oxypinakon die Formel $\mathrm{C}_{28} \mathrm{H}_{26} \mathrm{O}_{4}$ zukäme. Die Aufklärung der Konstitution dieser Verbindung ist mir bislang nicht gelungen.

Durch Zink und Salzsäure oder durch Zink und Kalilauge wird $p$-Oxydesoxybenzoin nicht reduziert, so daß die Darstellung der entsprechenden Oxypinakone nicht möglich war.

Außer dem Oxydations- und Reduktionsprodukte des $p$-Oxydesoxybenzoins habe ich zwei Halogensubstitutionsprodukte dargestellt, und zwar durch Einführung von Brom das Monobrom-p-Oxydesoxybenzoin und durch Einführung von Jod das Monojod- $p$-Oxydesoxybenzoin.

In beiden Fällen erfolgt der Eintritt des Halogens in der Seitenkette, so daß folgendes Formelbild die beiden Halogenderivate kennzeichnet:

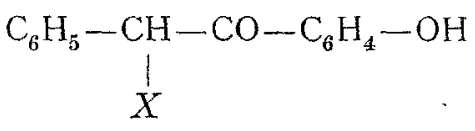

Der Eintritt des Halogens in der Seitenkette ist leicht nachzuweisen, indem beide Halogenderivate, in alkoholischer Lösung mit Silbernitratiösung versetzt, in der Kälte schon quantitative Abscheidung des Halogens als Halogensilber geben.

Eingangs erwähnte ich, daß bei der Kondensation von Säuren mit Phenolen sich unter gewissen Umständen nicht das Oxyleton bildet, sondern daß auch die Bildung von Phenylestern möglich ist.

Da der Phenylester der Phenylessigsäure in der Literatur nicht beschrieben ist, beschloß ich, seine Darstellung nach der Reaktionsgleichung zu versuchen:

$$
\begin{aligned}
\mathrm{C}_{6} \mathrm{H}_{5}-\mathrm{CH}_{2}-\mathrm{COO} \mathrm{H}+\mathrm{OH} & -\mathrm{C}_{6} \mathrm{H}_{5}= \\
& =\mathrm{C}_{6} \mathrm{H}_{5}-\mathrm{CH}_{2}-\mathrm{COOC}_{6} \mathrm{H}_{5}+\mathrm{H}_{2} \mathrm{O} .
\end{aligned}
$$


Auch diese Synthese ist mir gelungen. Die Esterifikation von Phenolen mit Säuren ist bekanntlich nicht so leicht durchführbar, wie jene von Alkoholen und Säuren, weil ja die Phenole einen ausgeprägt sauren Charakter haben.

Die gewöhnlichen Methoden, wie Esterifikation mit alkoholischer Salzsäure, Salzsäuregas oder konzentrierter Schwefelsäure lassen hier im Stich und man hat meist das Phosphoroxychlorid zu derartigen Esterifikationen verwendet. In meinem Falle führte auch dieses Mittel nicht zum Ziele, indem Phosphoroxychlorid vollständige Verharzung herbeiführte.

Bakunin ${ }^{1}$ hat vor nicht langer Zeit die Synthese des Phenylesters der Monochloressigsäure unter Zuhilfenahme von Phosphorpentoxyd durchgeführt. Da die Phenylessigsäure eine ganz ähnliche Zusammensetzung wie Monochloressigsäure in der Hinsicht zeigt, daß in beiden Substanzen ein Wasserstoffatom der Essigsäure durch eine negative Gruppe ersetzt ist, so schien mir die Esterifikation der Phenylessigsäure mit Phosphorpentoxyd aussichtsreich. Auf diesem Wege ist mir die Synthese denn auch gelungen unter Verwendung von Chloroform als Verdünnungsmittel.

Die Kondensation geht sehr glatt schon beim Siedepunkte des Chloroforms, $61.2^{\circ}$, vor sich und sind auch die Ausbeuten befriedigend $z u$ nennen.

Diese in der Literatur noch wenig bekannte Reaktion dürfte für die Herstellung von Phenylestern allgemeine Gültigkeit haben.

Der Phenylessigsäurephenylester ist fest, besitzt aber den niedrigen Schmelzpunkt von $35^{\circ}$. Den Beweis für die Konstitution habe ich durch Spaltung mit Kalilauge nach der Reaktionsgleichung:

$\mathrm{C}_{6} \mathrm{H}_{5}-\mathrm{CH}_{2}-\mathrm{COOC}_{6} \mathrm{H}_{5}+\mathrm{H}_{2} \mathrm{O}=$

erbracht.

$$
=\mathrm{C}_{6} \mathrm{H}_{5}-\mathrm{CH}_{2}-\mathrm{COOH}+\mathrm{C}_{6} \mathrm{H}_{5} \mathrm{OH}
$$

Mit dem $p$-Oxydesoxybenzoin ist der Phenylessigsäurephenylester natürlich isomer.

1 Gazzetta chimica, 30 (2). 358 


\section{B. Experimenteller Teil.}

\section{Darstellung des $p$-Oxydesoxybenzoins aus Phenylessig- säure und Phenol.}

$20 \mathrm{~g}$ fein gepulverte Phenylessigsäure wurden mit $14 \mathrm{~g}$ Phenol in einem Kolben auf dem Sandbade zusamniengeschmolzen und die farblose Flüssigkeit weiter zum schwachen Sieden am Rückflußkühler erhitzt. Nach und nach wurden nun in kleinen Portionen $60 \mathrm{~g}$ frisch umgeschmolzenes und gepulvertes Zinkchlorid in die Flüssigkeit eingetragen.

Man kann auch größere Mengen Zinkchlorid als jene, die dem dreifachen Gewichte der Phenylessigsäure gleichkommen, verwenden, erhält jedoch keine wesentlich besseren Ausbeuten.

Schon die ersten Partien des eingetragenen Zinkchlorids rufen eine braune Färbung der Flüssigkeit hervor, die beim weiteren Zusatz von Zinkchlorid immer intensiver wird. Die Reaktionstemperatur liegt bei $170^{\circ}$ bis $200^{\circ}$.

$\mathrm{Da}$ nach jedesmaligem Eintragen von Zinkchlorid die Temperatur steigt, ist es vorteilhaft, nach dem Eintragen immer die Flamme unter dem Sandbade zu entfernen, da über $220^{\circ}$ Verharzung eintritt.

Die Flüssigkeit wird immer dickflüssiger; in einer Stunde ist alles Zinkchlorid eingetragen. Man erhitzt noch eine weitere halbe Stunde zum schwachen Sieden und läßt dann erkalten.

Die Reaktionsflüssigkeit erstarrt zu einer braunroten, zähen Masse; sie wurde mit Benzol und ein wenig Wasser behandelt. Die dunkelrot gefärbte Benzollösung wird alsdann im Scheidetrichter so oft mit ein wenig mit Salzsäure angesäuertem Wasser geschüttelt, bis alles Zinkchlorid entfernt ist. Würde man auf einmal viel Wasser zur Benzollösung bringen, so würde das in Wašser unlösliche Reaktionsprodukt in gelatinöser, schwer filtrierbarer Form ausfallen.

Die vom Zinkchlorid befreite Auflösung der Reaktionsmasse in Benzol wird nun im Scheidetrichter mit kalt ge- 
sättigter Natriumcarbonatlösung so oft ausgeschüttelt, als noch rotviolette Färbung der Sodalösung eintritt.

$\mathrm{Da}$ das $p$-Oxydesoxybenzoin eine phenolische Hydroxylgruppe trägt, war die Löslichkeit in alkalischen Flüssigkeiten vorauszusehen. Laugen selbst zu verwenden ist nicht angezeigt, da bei der Kondensation viel unangegriffenes Phenol verblieb, welches als Phenolat in Lösung gegangen wäre, so daß eine Trennung vom Reaktionsprodukt unmöglich gewesen wäre.

Die vereinigten alkalischen Flüssigkeiten werden alsdann mit Salzsäure $(D=1 \cdot 19)$ bis zur schwach sauren Reaktion versetzt, wobei ein hochrot gefärbter, kristallinischer Körper ausfällt, dessen Menge sich beim Erkalten der Flüssigkeit noch bedeutend vermehrt.

Der entstandene Niederschlag wird abgesaugt und mit kaltem Wasser gewaschen, um mitausgefallene, unveränderte Phenylessigsäure $z \mathrm{u}$ entfernen. Der Rückstand wird aus viel siedendem Wasser mehrmals umkristallisiert, wobei das $p$-Oxydesoxybenzoin in gelbrötlichen, federartigen Kristallen beim Erkalten ausfällt.

Das p-Oxydesoxybenzoin ist in kaltem Wasser unlöslich, in heißem löst sich 1 Teil in zirka 150 Teilen Wasser. In Alkalien ist es leicht, in Säuren unlöslich. Leicht löslich ist das Produkt ferner in Eisessig, Alkohol, Äther und Benzol. Zum Umkristallisieren eignet sich am besten Wasser.

Der Schmelzpunkt liegt bei $142^{\circ}$ (korr.).

Mit Eisenchloridlösung gibt das p-Oxydesoxybenzoin eine charakteristische Reaktion, indem 1 Teil $p$-Oxydesoxybenzoin in 10 Teilen Alkohol gelöst, mit wässeriger Eisenchloridlösung (1:10) versetzt, eine dunkelbraune Färbung gibt.

Die Analyse der bei $100^{\circ}$ getrockneten Substanz ergab nachfolgende Werte:

I. $0 \cdot 1993 \mathrm{~g}$ Substanz gaben $0.5781 \mathrm{~g}$ Kohlensäure und $0.0981 \mathrm{~g}$ Wasser.

II. $0 \cdot 2001 \mathrm{~g}$ Substanz gaben $0.5808 \mathrm{~g}$ Kohlensäure und $0 \cdot 1003 \cdot g$ Wasser. 
In 100 Teilen:

\begin{tabular}{|c|c|c|c|}
\hline & \multicolumn{2}{|c|}{ Gefunden } & $\begin{array}{r}\text { Berechnet für } \\
\mathrm{C}_{14} \mathrm{H}_{12} \mathrm{O}_{2}\end{array}$ \\
\hline & I. & II. & \\
\hline & $79 \cdot 11$ & $79 \cdot 16$ & $79 \cdot 24$ \\
\hline 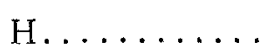 & $5 \cdot 47$ & $5 \cdot 57$ & $5 \cdot 66$ \\
\hline
\end{tabular}

\section{Darstellung des $p$-Oxydesoxybenzoins aus Phenylessig- säurechlorid und Phenol.}

$20 \mathrm{~g}$ Phenylessigsäurechlorid und $12 \cdot 2 \mathrm{~g}$ Phenol wurden in $60 \mathrm{~g}$ Nitrobenzol in einem Kolben gelöst, die Lösung auf dem Wasserbade auf $80^{\circ}$ erwärmt und nun nach und nach $37 \mathrm{~g}$ feingepulvertes Aluminiumchlorid in kleinen Partien eingetragen.

Nach jedesmaligem Eintragen erfolgt Aufschäumen und Entweichen von Salzsäure. Die Reaktionstemperatur von $80^{\circ}$ genügt zur Vollendung der Reaktion und ist eine höhere Temperatur nicht nötig.

In je gemäßigteren Grenzen man den Verlauf der Reaktion leitet, um so weniger harzige Produkte entstehen. Dies wird dadurch erreicht, daß man besonders anfangs nur sehr kleine Mengen Aluminiumchlorid (etwa $0.5 \mathrm{~g}$ ) einträgt und immer wartet, bis die Flüssigkeit nicht mehr schäumt. Später kann das Eintragen rascher erfolgen, da die Reaktion gegen Ende träge verläuft.

Im Verlaufe einer halben Stunde ist alles Aluminiumchlorid eingetragen; die Flüssigkeit hat eine dunkelrote Färbung angenommen. Man erwärmt am Schlusse eine Viertelstunde auf dem Wasserbade, bis die Salzsäureentwicklung nachgelassen hat, läßt dann auf $30^{\circ}$ erkalten und gießt das sirupöse Reaktionsprodukt in $300 \mathrm{~cm}^{3}$ Wasser ein.

Unter Zusatz einiger Tropfen Salzsäure erwärmt man kurze Zeit über freier Flamme, um alles Aluminiumchlorid in Lösung zu bringen. Nun lässt man ganz erkalten, trennt die Nitrobenzolschichte von der wässerigen Schichte, nimmt erstere 
sodann in Benzol auf und wäscht das restliche Aluminium. chlorid durch wiederholten Zusatz kleiner Mengen Wassers aus. Der Zusatz größerer Mengen Wassérs auf einmal muß unterbleiben, weil sonst das in Wasser unlösliche Reaktionsprodukt gelatinös ausfällt.

Die rot gefärbte Benzollösung wird nun so lange mit wässeriger Natronlauge $(D=1 \cdot 22)$ ausgeschüttelt, als diese Lösung noch rotviolett gefärbt wird.

Bei dieser Synthese ist die Verwendung von Natriumcarbonatlösung nicht notwendig, da kein unangegriffenes Phenol vorhanden ist. Die Verwendung von Natronlauge erscheint deshalb angebracht, weil das Reaktionsprodukt in Natronlauge leichter als in Natriumcarbonat löslich ist.

Die vereinigten alkalischen Flüssigkeiten werden mit Salzsäure $(D=1 \cdot 19)$ bis zur schwach sauren Reaktion versetzt, wobei ein brauner, kristallinischer Niederschlag ausfällt.

Man filtriert nach dem Erkalten, wäscht mit Wasser und erhält so das Rohprodukt in Ausbeuten, die bis zu $90 \%$ der Theorie entsprechen.

Einmal aus Wasser umkristallisiert, nimmt der Niederschlag die hochrote Farbe an, ähnlich wie sie bei dem nach dem ersten Verfahren dargestellten Produkte zu sehen war.

Weiteres zweimaliges Umkristallisieren aus Wasser gibt reines $p$-Oxydesoxybenzoin von gleichem Aussehen und den gleichen Eigenschaften wie das nach dem ersten Verfahren erhaltene.

Die Ausbeute an reinem $p$-Oxydesoxybenzoin enspricht 60 bis $70 \%$ der Theorie gegenüber $20 \%$ beim ersten Verfahren.

Hauptbedingungen für das Gelingen der Reaktion sind, wie mich mehrfache Versuche gelehrt haben, die Verwendung von absolut trockenem Aluminiumchlorid und gemäßigter Yerlauf des Darstellungsprozesses.

Die Analyse der bei $100^{\circ}$ getrockneten Substanz ergab nachfolgende Werte:

$0.2004 g$ Substanz gaben $0.5829 g$ Kohlensäure und $0 \cdot 1001 \mathrm{~g}$ Wasser. 
In 100 Teilen:

\begin{tabular}{|c|c|c|}
\hline & Gefunden & $\begin{array}{l}\text { Berechnet für } \\
\mathrm{C}_{14} \mathrm{H}_{12} \mathrm{O}_{2}\end{array}$ \\
\hline & $79 \cdot 33$ & $79 \cdot 24$ \\
\hline $\mathrm{H}$. & $5 \cdot 55$ & $5 \cdot 66$ \\
\hline
\end{tabular}

3. Molekulargewichtsbestimmung des $p$-Oxydesoxybenzoins.

Die Molekulargewichtsbestimmung des $p$-Oxydesoxybenzoins wurde nach der Methode der Siedepunktserhöhung in: dem von Beckmann angegebenen Apparate durchgeführt. Als. Lösungsmittel wurde Eisessig verwendet.

Ich erhielt als Molekulargewicht 205 gegenüber dem berechneten Werte 212.

\section{Darstellung des Acetylproduktes des p-Oxydesoxy- benzoins.}

Um den Beweis für das Vorhandensein einer freien phenolischen Hydroxylgruppe im $p$-Oxydesoxybenzoin $\mathrm{C}_{6} \mathrm{H}_{5}-\mathrm{CH}_{2}-\mathrm{CO}-\mathrm{C}_{6} \mathrm{H}_{4} \mathrm{OH}$ zu erbringen, habe ich versucht, ein Acetylderivat herzustellen.

$2 g p$-Oxydesoxybenzoin werden in der zehnfachen Menge Essigsäureanhydrid in der Wärme gelöst und zwei Stunden am Rückflußkühler zum schwachen Sieden über freier Flamme erhitzt.

Die Reaktionsflüssigkeit wird alsdann zur Entfernung des. überschüssigen Essigsäureanhydrids auf dem Wasserbade mehmals mit Methylalkohol abgedampft. Es bleibt ein hellgelbes Öl zurück, das in der Kälte rasch zu großen, sechsseitigen Tafeln erstarrte. Zur Entfernung von etwa vorhandenem p-Oxydesoxybenzoin wurden diese Kristalle mit $200 \mathrm{~cm}^{3}$ verdünnter Natriumcarbonatlösung ausgekocht und heiß filtriert.

Das am Filter verbleibende Öl wurde in kaltem 95 prozentigen Alkohol gelöst, filtriert und in $500 \mathrm{~cm}^{3}$ kaltes Wasser eingetragen. Aus der entstandenen Emulsion scheidet sich beim kurzen Stehen das Acetylprodukt in kleinen, geiblichweißen Täfelchen ab. Die Kristalle wurden abgesaugt, durch 
Waschen mit kaltem Wasser vom Alkohol befreit. Nach zweimaligem Umkristallisieren blieb der Schmelzpunkt konstant bei $82^{\circ}$ (korr.).

Das Acetylprodukt des $p$-Oxydesoxybenzoins ist in Wasser unlöslich, schwer löslich in Alhohol, leichter in Äther.

Es gibt keine Farbenreaktionen.

Die Ausbeute betrug $90 \%$ der Theorie.

Die Analyse der über Schwefelsäure getrockneten Substanz ergab nachfolgende Werte:

1. $0.2317 \mathrm{~g}$ Substanz gaben $0.6413 \mathrm{~g}$ Kohlensäure und $0 \cdot 1170 \mathrm{~g}$ Wasser.

II. $0.2060 \mathrm{~g}$ Substanz gaben nach der Verseifung mit verdünnter Schwefelsäure Essigsäure ${ }^{1}$, welche $8 \cdot 2 \mathrm{~cm}^{3}$ Kalilauge vom Titer $0.005609 \mathrm{~g}$ KHO zur Neutralisation brauchte.

In 100 Teilen:

\begin{tabular}{|c|c|c|c|}
\hline & \multicolumn{2}{|c|}{ Gefunden } & \multirow{2}{*}{$\begin{array}{c}\text { Berechnet für } \\
\mathrm{C}_{6} \mathrm{H}_{5}-\mathrm{CH}_{2}-\mathrm{CO}-\mathrm{C}_{6} \mathrm{H}_{4} \mathrm{O}-\mathrm{C}_{2} \mathrm{H}_{3} \mathrm{O}\end{array}$} \\
\hline & I & II & \\
\hline$\ldots$ & $75 \cdot 49$ & - & $75 \cdot 55$ \\
\hline & $5 \cdot 61$ & - & $5 \cdot 56$ \\
\hline $\mathrm{C}_{2} \mathrm{H}_{3} \mathrm{O} \ldots$ & - & $17 \cdot 11$ & $16 \cdot 93$ \\
\hline
\end{tabular}

\section{Darstellung des Oxims des $p$-Oxydesoxybenzoins.}

Das $p$-Oxydesoxybenzoin kann, wenn ihm die angesprochene Konstitution $\mathrm{C}_{6} \mathrm{H}_{5}-\mathrm{CH}_{2}-\mathrm{CO}-\mathrm{C}_{6} \mathrm{H}_{4} \mathrm{OH}$ zukommt, ein Oxim geben, da ein doppelt gebundenes Sauerstoffatom vorhanden ist.

Die Oximierung wurde analog der von V. Meyer und Oelkers ${ }^{2}$ zur Darstellung des Desoxybenzoinoxims verwendeten Methode ausgeführt.

$2 g p$-Oxydesoxybenzoin wurden in $50 g 95$ prozentigem Alkohol gelöst und mit einer. Auflösung von $0.65 g$ salzsaurem

1 Monatshefte für Chemie, $18,659$.

2 Berl. Ber., 21, 1295. 
Hydroxylamin und der äquivalenten Menge von Natriumcarbonat $(0.5 \mathrm{~g})$ in $10 \mathrm{~cm}^{3}$ Wasser versetzt und am Rückflußkühler auf dem Wasserbade mehrere Stunden erhitzt.

Die Oximierung geht sehr langsam vor sich und es ist zweckmäßig, nach 2 bis 3 Stunden eine kleine Menge Hydroxylaminchlorhydrat und die äquivalente Menge Natriumcarbonat, in ein wenig Wasser gelöst, zur Flüssigkeit hinzuzusetzen.

Wiederholt man nach einigen Stunden diese Operation neuerdings und erhitzt weitere 3 Stunden, so findet fast quantitative Umsetzung zu Oxim statt.

Dampft man alsdann die Reaktionsflüssigkeit auf dem Wasserbade $a b$, so hinterbleibt ein gelbes Öl, das nur schwer bei Zimmertemperatur, leicht aber bei $8^{\circ}$ bis $9^{\circ} \mathrm{zu}$ einer kristallinischen Masse erstarrt.

Dieses Reaktionsprodukt wird mit Wasser gewaschen, um das enstandene Kochsalz zu entfernen, sodann in möglichst wenig kaltem 95 prozentigem Alkohol gelöst und in $100 \mathrm{~cm}^{3}$ Wasser eingetragen. Aus der entstandenen Emulsion scheidet sich nach einiger Zeit das $p$-Oxydesoxybenzoinoxim in glänzenden, gelblichweißen Kristallen $a b$, die abgesaugt und mit kaltem Wasser gewaschen werden.

Auf diese Weise zweimal umkristallisiert, bleibt der Schmelzpunkt des Oxims konstant bei $85^{\circ}$ (korr.).

Das $p$-Oxydesoxybenzoinoxim ist im Wasser sehr schwer, leichter in Alkohol löslich. Löslich ist es auch in Eisessig und Äther.

Die Analyse der über. Schwefelsäure getrockneten Substanz ergab nachfolgende Werte:

I. $0.2043 \mathrm{~g}$ Substanz gaben $11 \cdot 5 \mathrm{~cm}^{3}$ Stickstoff bei $748 \mathrm{~mm}$ Druck und $16^{\circ} \mathrm{C}$.

II. $0.2018 \mathrm{~g}$ Substanz gaben $11.3 \mathrm{~cm}^{3}$ Stickstoff bei $741 \mathrm{~mm}$ Druck und $15^{\circ} \mathrm{C}$.

In 100 Teilen:

\begin{tabular}{|c|c|c|c|}
\hline \multirow{2}{*}{\multicolumn{3}{|c|}{$\overbrace{\mathrm{I}}^{\text {Gefunden }}$}} & \multirow{2}{*}{$\begin{array}{r}\text { Berechnet für } \\
\mathrm{C}_{14} \mathrm{H}_{13} \mathrm{NO}_{2}\end{array}$} \\
\hline & & & \\
\hline & $6 \cdot 45$ & $6 \cdot 38$ & $6 \cdot 18$ \\
\hline
\end{tabular}




\section{Spaltung des $p$-Oxydesoxybenzoins.}

Knoevenage ${ }^{1}$. und Arndts haben die Spaltung des Desoxybenzoins mit konzentrierter, wässeriger Kalilauge vorgenommen.

Ich versuchte die Spaltung des $p$-Oxydesoxybenzoins mit dem gleichen Agens und konnte auch ein positives Resultat erzielen.

$5 g p$-Oxydesoxybenzoin wurden in $15 \mathrm{~g} 70$ prozentiger wässeriger Kalilauge gelöst und 1 Stunde am Rückflußkühler erhitzt. Schon nach kurzer Zeit begann die Ausscheidung von Öltröpfchen, ein Zeichen, daß Spaltung eingetreten war.

Die erkaltete Flüssigkeit wurde im Scheidetrichter mit Äther behandelt. Die ätherische, mit Wasser mehrmals gewaschene Auflösung wurde über Calciumchlorid getrocknet und sodann der fraktionierten Destillation unterworfen.

Nach dem Verdampfen des Äthers auf dem Wasserbade blieb eine farblose, benzolartig riechende Flüssigkeit zurück, die bei der Destillation bei $110^{\circ}$ überging. Siedepunkt und sonstige Eigenschaften stimmen mit Toluol überein.

Die wässerige alkalische Lösung, die früher vom Äther abgelassen wurde, wurde mit verdünnter Salzsäure angesäuert, um die Oxybenzoesäure aus dem Kalisalz in Freiheit zu setzen.

Dann wurde neuerdings ausgeäthert, mit Wasser gewaschen und der Äther verdunstet. Die zurückbleibende kristallinische Masse wurde aus siedendem Wasser einmal umkristallisiert, wobei kleine, weiße Prismen erhalten wurden. Bei $100^{\circ}$ getrocknet, verloren die Kristalle Wasser und schmolzen dann bei $210^{\circ}$ (korr.).

Die Analyse der über Schwefelsäure getrockneten Substanz ergab nachfolgende Werte:

I. $0.2001 g$ Substanz gaben $0.3943 g$ Kohlensäure und $0.0915 \mathrm{~g}$ Wasser.

II. $0.2102 g$ Substanz gaben $0.4145 g$ Kohlensäure und $0.0963 g$ Wasser.

1 Berl. Ber., 35, 1982. 
In 100 Teilen:
$\overbrace{\mathrm{I}}^{\text {Gefunden }} \overbrace{\mathrm{II}}$
C......53:74 $53 \cdot 78$
Berechnet für
$\underbrace{\mathrm{C}_{6} \mathrm{H}_{4}(\mathrm{OH}) \cdot \mathrm{COOH} \cdot \mathrm{H}_{2} \mathrm{O}}$
$53 \cdot 84$
H...... $5.08 \quad 5.09$
$5 \cdot 13$

Die vorliegende Substanz war also $p$-Oxybenzoesäure und es erscheint somit die Konstitution des $p$-Oxydesoxybenzoins auch aus seinen Spaltungsprodukten sichergestellt.

\section{Darstellung des $p$-Oxybenzils durch Oxydation des $p$-Oxydesoxybenzoins.}

Wie ich im theoretischen Teile auseinandersetzte, konnte bei der Oxydation des $p$-Oxydesoxybenzoins zweierlei geschehen. Entweder es trat Spaltung in Benzoesäure und $p$-Oxybenzoesäure ein, oder zwei Wasserstoffatome in der Methylengruppe wurden oxydiert und es mußte $p$-Oxybenzil entstehen.

Da die Bildung des letztgenannten Körpers der interessantere Vorgang ist, so war ich von vornherein bestrebt, die Oxydationsbedingungen so $\mathrm{zu}$ gestalten, daß eine möglichst milde Einwirkung stattfände.

Deshalb nahm ich die Oxydation mit Chromsäure und Eisessig in sehr verdünnten Lösungen vor.

$1 g p$-Oxydesoxybenzoin wird in $50 \mathrm{~g}$ Eisessig gelöst und am Rückflußkühler zum Sieden erhitzt. In die siedende Flüssigkeit läßt man mittels eines langsam tropfenden Kapillarhebers, den man in das Kühlrohr von oben einführt, eine Auflösung von $0.626 \mathrm{~g}$ Chromtrioxyd in $30 \mathrm{~g}$ Eisessig einfließen. Diese Operation soll für $1 g$ Substanz 3 Stunden beanspruchen. Jeder einfallende Tropfen erzeugt sogleich eine smaragdgrüne Färbung der Flüssigkeit.

Ist die Oxydation beendigt, so läßt man erkalten, trägt dann die tiefgrün gefärbte Flüssigkeit in $400 \mathrm{~cm}^{2}$ Wasser ein und setzt Ammoniak zur teilweisen Abstumpfung der Essigsäure zu; dabei soll die Flüssigkeit sauer bleiben.

Das Oxydationsprodukt fällt sogleich in großen, gelben Flocken aus, die einen mandelartigen Geruch besitzen. Dies 
ist wahrscheinlich darauf zurückzuführen, daß ein Teil des $p$-Oxydesoxybenzoins zu Benzaldehyd oxydiert wurde.

Der schwer zu filtrierende Niederschlag wird abgesaugt und mit Salzsäure $(1: 10)$ solange gewaschen, bis alles Chrom entfernt ist, worauf man die Salzsäure durch Wasser verdrängt. Der Rückstand wird in kaltem 95 prozentigem Alkohol gelöst, filtriert und mit Wasser ausgefällt. Diese Operation wiederholt man 2 bis 3 mal.

Man kann das Fällen und Wiederauflösen auch aus Eisessig vornehmen und hat dann nach gutem Auswaschen mit Wasser amorphes, aber analysenreines $p$-Oxybenzil.

Das $p$-Oxybenzil ist nur äußerst schwierig kristallisiert $\mathrm{zu}$ erhalten, da es einerseits in Wasser ganz unlöslich ist, andererseits in organischen Lösungsmitteln sich sehr leicht auflöst; aus letzteren Lösungsmitteln fällt es pulverig aus, beim Verdünnen mit Wasser flockig oder schleimig.

Auch zeigt sich der Übelstand, daß das $p$-Oxybenzil beim Erwärmen in seinen Lösungsmitteln schmilzt.

Beim wochenlangen Stehen einer kalt gesättigten, mit etwas Wasser versetzten alkoholischen Lösung fällt ein Teil in schönen, orangefarbigen, kleinen Nadeln aus. Hierbei geht aber viel Material verloren.

Die amorphe und die kristallisierte Substanz schmilzt bei $175^{\circ}$ (korr.).

Die Ausbeute beträgt $80 \%$ der Theorie.

Die Analyse der über Schwefelsäure getrockneten Substanz ergab nachfolgende Werte:

I. $0 \cdot 2501 \mathrm{~g}$ Substanz gaben $0.6808 \mathrm{~g}$ Kohlensäure und $0 \cdot 1028 g$ Wasser;

II. $0 \cdot 2008 g$. Substanz gaben $0 \cdot 5464 g$ Kohlensäure und $0.0819 \mathrm{~g}$ Wasser.

In 100 Teilen:

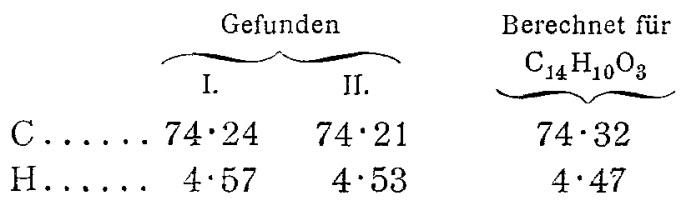


Den Nachweis der beiden Ketogruppen im p-Oxybenzil habe ich zunächst qualitativ mit Hilfe der von E. Bamberger ${ }^{1}$ angegebenen Farbenreaktion für $\alpha$-Diketone erbracht.

1 Teil $p$-Oxybenzil wird in 5 Teilen 95 prozentigem Alkohol in der Kälte gelöst, wobei die Farbe der Flüssigkeit hellgelb ist. Trägt man nun ein erbsengroßes Stückchen Ätzkali in die Flüssigkeit ein, so entsteht sofort eine tief dunkelrote Färbung, die beim Erhitzen verschwindet.

Die beiden Ketogruppen habe ich ferner durch Darstellung eines Dioxims nachgewiesen.

\section{Darstellung des $p$-Oxybenzildioximchlorhydrates.}

Die Dioximierung des p-Oxybenzils habe ich ähnlich ausgeführt, wie es die von V. Meyer und Auwers ${ }^{2}$ für die Darstellung des Dioxims des Benzils verwendete Methode vorschreibt.

$2 g p$-Oxybenzil werden in $50 \mathrm{~g} 95$ prozentigem Alkohol gelöst, mit der berechneten Menge von $(1 \cdot 22 g)$ Hydroxylaminchlorhydrat versetzt, wobei man das salzsaure Hydroxylamin in gepulverter Form einträgt; nun fügt man einige Tropfen konzentrierte Salzsäure hinzu und erhitzt mehrere Stunden am Rückflußkühler auf dem Wasserbade zum Sieden.

Die Oximierung geht hier am besten in saurer Lösung vor sich.

Da die Bildung des Dioxims, ähnlich wie beim $p$-Oxydesoxybenzoinoxim, nur langsam vor sich geht, so ist es vorteilhaft, in Zwischenräumen von 2 bis 3 Stunden neuerdings kleine Mengen von gepulvertem Hydroxylaminchlorhydrat und einige Tropfen Salzsäure zur Flüssigkeit hinzuzusetzen.

Nach 8 bis 10 Stunden bringt man die. Flüssigkeit in eine Abdampfschale und dampft auf dem Wasserbade zur Trockene ein.

Hierbei scheiden sich zwei Bestandteile ab; unverändertes, braunes $p$-Oxybenzil und weiße, prismatische Nädelchen.

1 Berl. Ber. 18, 865. Vergl. Lie big's Annalen 17, 91 .

2 Berl. Ber. 21, 792. 
Der erkaltete Trockenrückstand wird mit Eisessig digeriert, wodurch das $p$-Oxybenzil in Lösung gebracht wird, während die Nädelchen unverändert zurückbleiben; man filtriert und wäscht so lange mit Eisessig, bis die Nädelchen rein weiß erscheinen. Das so erhaltene Rohprodukt wird in absoluten Alkohol in der Wärme gelöst und filtriert. Beim langsamen Erkalten der Flüssigkeit scheiden sich 2 bis $3 \mathrm{~cm}$ lange, weiße prismatische Nadeln ab, die mit kaltem, absoluten Alkohol gewaschen werden und die, getrocknet bei $155^{\circ}$ (korr.), unter Zersetzung schmelzen.

Das $p$-Oxybenzildioximchlorhydrat ist in kaltem, absoluten Alkohol schwer, in warmem leicht löslich. Sehr leicht löslich ist es in Wasser und verdünntem Alkohol. Ganz unlöslich ist es in Eisessig.

Die Ausbeute betrug 80 bis $90 \%$ der Theorie.

I. $0 \cdot 1987 \mathrm{~g}$ Substanz gaben $14 \cdot 8 \mathrm{~cm}^{3}$ Stickstoff bei $13^{\circ} \mathrm{C}$. und $748 \cdot 3 \mathrm{~mm}$ Druck.

II. $0 \cdot 2137 \mathrm{~g}$ Substanz gaben $16 \cdot 3 \mathrm{~cm}^{3}$ Stickstoff bei $15^{\circ} \mathrm{C}$. und $743 \cdot 5 \mathrm{~mm}$ Druck.

III. $0 \cdot 2037 \mathrm{~g}$ Substanz gaben bei der Halogenbestimmung mit Ätzkalk $0 \cdot 1787 \mathrm{~g}$ Chlorsilber.

In 100 Teilen:

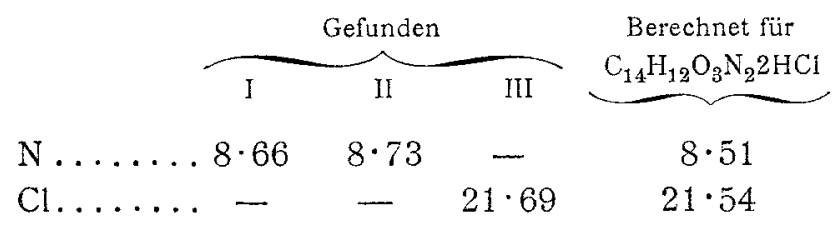

\section{Darstellung des Reduktionsproduktes des p-Oxydesoxy- benzoins.}

Unter der Annahme, daß die Reduktion unter Bildung von Oxytoluylenhydrat verlaufen würde, löste ich $3 g p$-Oxydesoxy benzoin in $60 \mathrm{~g} 95$ prozentigem Alkohol, erhitzte auf dem Wasserbade am Rückflußkühler zum Sieden, wobei Kohlendioxyd eingeleitet wurde und trug $26 \mathrm{~g}$ fünfprozentiges Natriumamalgam, entsprechend der doppelten theoretischen 
Menge Natriums (1.32g), in kleinen Portionen in die Flüssigkeit ein.

Das Einleiten des Kohlendioxyds bezweckte, die Flüssigkeit dauernd neutral zu halten.

Schon nach kurzer Zeit beginnt die Ausscheidung von Natriumcarbonat. In 3 Stunden war alles Amalgam eingetragen; es wurde erkalten gelassen und filtriert. Das Natriumcarbonat am Filter wurde mit Alkohol ausgewaschen.

Das schwach alkalische, rotviolette Filtrat wurde sodann mit Salzsäure $(D=1.08)$ schwach angesäuert und mit $100 \mathrm{~cm}^{3}$ Wasser versetzt. Nach dem Ansäuern war die Flüssigkeit gelb gefärbt.

Aus der entstandenen Emulsion fiel beim teilweisen Verdampfen des Alkohols auf dem Wasserbade das Reduktionsprodukt zum Teil ölig, zum Teil in gelben, glänzenden Blättchen aus. Die ölige, von Kristallen durchsetzte Masse wurde nun filtriert, die Kristalle mit angesäuertem Wasser gewaschen, in möglichst wenig kaltem 95 prozentigen Alkohol gelöst und die Lösung in viel Wasser $\left(500 \mathrm{~cm}^{3}\right)$ eingetragen. Das Reduktionsprodukt fällt hierbei in großen, gelben Flocken aus.

Das so gereinigte Produkt wurde nach dem Filtrieren und Waschen mit Wasser neuerdings in 95prozentigem Alkohol gelöst, mit etwas Wasser verdünnt und nun auf dem Wasserbade auf $75^{\circ}$ erwärmt.

Das Reduktionsprodukt fällt nach längerer Zeit in kleinen Kristallblättchen aus, doch darf man die angegebene Temperatur nicht überschreiten, weil sonst ein Teil wieder ölig fällt; man filtriert und wäscht mit Wasser. Das so erhaltene Produkt ändert bei weiteren Umkristallisationen auf die nämliche Weise den Schmelzpunkt nicht mehr; es schmilzt bei $165^{\circ}$ (korr.).

Das Reduktionsprodukt ist in Wasser fast unlöslich, in kaltem Alkohol schwer, leicht in warmem Alkohol, Eisessig und Äther löslich.

Aus $3 g p$-Oxydesoxybenzoin wurden $2 \cdot 2 g$ Reduktionsprodukt erhalten.

Die nachfolgend angegebenen Werte der Analysen zeigen, daß das Reduktionsprodukt weder mit dem erwarteten Oxy- 
toluylenhydrat noch mit einem theoretisch möglichen Pinakon in den Zahlen Übereinstimmung zeigt.

Wie schon im theoretischen Teile erwähnt wurde, habe ich das $p$-Oxydesoxybenzoin mit Zink und Salzsäure und mit Zink und Kalilauge zu reduzieren versucht, habe aber unverändertes Ausgangsmaterial erhalten.

Für die Analyse wurde die Substanz bei $100^{\circ}$ getrocknet.

I. $0.2019 \mathrm{~g}$ Substanz gaben $0.6107 \mathrm{~g}$ Kohlensäure und $0 \cdot 1137 g$ Wasser.

II. $0.2122 g$ Substanz gaben $0.6415 g$ Kohlensäure und $0.1186 g$ Wasser.

In 100 Teilen:

\begin{tabular}{|c|c|c|}
\hline \multicolumn{2}{|c|}{ Gefunden } & Berechnet fün \\
\hline & II. & $\mathrm{C}_{14} \mathrm{H}_{14} \mathrm{O}_{2}$ \\
\hline $.82 \cdot 48$ & $82 \cdot 45$ & $78 \cdot 45$ \\
\hline$\ldots \ldots \ldots 6 \cdot 26$ & $6 \cdot 21$ & $6 \cdot 60$ \\
\hline
\end{tabular}

Das Reduktionsprodukt hat also einen bedeutend größeren Kohlenstoffgehalt und kommt ihm die einfachste Formel $\mathrm{C}_{29} \mathrm{H}_{27} \mathrm{O}_{3}$ zu.

\section{Darstellung des Monobrom- $p$-oxydesoxybenzoins.}

$1 g$-Oxydesoxybenzoin wird in $50 g$ Eisessig gelöst und in die Lösung bei gewöhnlicher Temperatur aus einer Bürette $0.24 \mathrm{~cm}^{3}$ Brom, entsprechend $0.755 \mathrm{~g}$ einfließen gelassen.

Die rotbraun gefärbte Flüssigkeit wird nun auf dem Wasserbade bis auf $80^{\circ}$ erwärmt. Bei dieser Temperatur tritt Entfärbung unter Entweichen des Bromwasserstoffes auf.

Ist der Bromwasserstoff verjagt, so gießt man die erkaltete Lösung in das zehnfache Volumen Wasser. Alsbald fällt das Bromprodukt als rötliches öl aus.

Man filtriert, wäscht mit kaltem Wasser, löst das Öl in überschüssigem 95 prozentigen Alkohol, filtriert und läßt dann in viel Wasser einfließen.

Nach ein- bis zweitägigem Stehen fällt das Monobrom$p$-oxydesoxybenzoin in kristallinischer Form aus.

Beim Verdunsten einer alkoholischen Lösung scheidet sich das Monobrom-p-oxydesoxybenzoin ölig ab. Es ist in 
Wasser unlöslich, leicht löslich in Alkohol und Eisessig. Das ölig abgeschiedene Produkt erstarrt auch beim tagelangen Stehen nicht.

Das Monobrom- $p$-oxydesoxybenzoin schmilzt bei $108^{\circ}$ (korr.); die Ausbeute ist fast quantitativ.

Die Halogenbestimmung, welche durch Glühen der Substanz mit Ätzkalk und Behandlung des Halogencalciums nach der Methode Vortmann ausgeführt wurde, ergab für die über Schwefelsäure getrocknete Substanz nachfolgende Werte:

I. $0 \cdot 3072 \mathrm{~g}$ Substanz gaben $0 \cdot 1993 \mathrm{~g}$ Bromsilber.

II. $0 \cdot 3123 \mathrm{~g}$ Substanz gaben $0 \cdot 2020 \mathrm{~g}$ Bromsilber.

In 100 Teilen:

$\overbrace{\mathrm{I}}^{\mathrm{Br} \ldots .27 \cdot 61} \underbrace{\text { Gefunden }}_{\mathrm{II}} \underbrace{\begin{array}{c}\text { Berechnet für } \\ \mathrm{C}_{14} \mathrm{H}_{11} \mathrm{BrO}_{2}\end{array}}_{27 \cdot 53}$

Um den Beweis zu führen, daß der Eintritt des Broms in die Methylengruppe erfolgt ist, wurden $0.3001 \mathrm{~g}$ Monobrom$p$-oxydesoxybenzoin in wenig kaltem 95 prozentigen Alkohol gelöst und mit überschüssiger, wässeriger Silbernitratlösung versetzt.

Das gefällte Silberbromid wurde mit verdünntem Alkohol gewaschen und in üblicher Weise gewichtsanalytisch bestimmt.

$0 \cdot 3001 g$ der über Schwefelsäure getrockneten Substanz gaben $0 \cdot 1967 g$ Bromsilber.

In 100 Teilen:

$$
\text { Br....... } \underbrace{\text { Gefunden }}_{27 \cdot 89} \frac{\begin{array}{c}
\text { Berechnet für } \\
\mathrm{C}_{14} \mathrm{H}_{11} \mathrm{BrO}_{2}
\end{array}}{27 \cdot 47}
$$

\section{Darstellung des Monojod-p-oxydesoxybenzoins.}

Für die Darstellung des Monojod-p-oxydesoxydbenzoins habe ich die von Classen ${ }^{1}$ neuerdings empfohlene Methode der Jodierung in Boraxlösung mit bestem Erfolge verwendet.

D. R. P. 86069 , Berl. Ber. $28,1605$. 
$2 g p$-Oxydesoxybenzoin und $9 g$ Borax - entsprechend der fünffachen theoretischen Menge - werden in $300 \mathrm{~cm}^{3}$ siedendem Wasser gelöst und in die Auflösung eine Lösung von $2.4 \mathrm{~g}$ Jod in $2.7 \mathrm{~g}$ Jodkalium und $15 \mathrm{~cm}^{3}$ Wasser einfließen gelassen.

Die Jodjodkaliumlösung wird sofort entfärbt und es resultiert eine gelbgrün gefärbte Flüssigkeit, aus der sich nach kurzer Zeit das Monojod-p-oxydesoxybenzoin in Form eines bräunlichen, schweren, pulverigen Niederschlages abscheidet.

Der filtrierte, mit verdünnter Jodkaliumlösung und Wasser gewaschene Niederschlag wird in möglichst wenig warmem 95 prozentigen Alkohol gelöst und in $500 \mathrm{~cm}^{3}$ Wasser eingetraigen.

Das Monojod- $p$-oxydesoxybenzoin scheidet sich sogleich in rötlichweißen, großen Flocken ab.

Man filtriert, wäscht mit Wasser und fällt nochmals den Niederschlag nach dem Lösen in Alkohol mit Wasser aus.

Das so gereinigte Monojod- $p$-oxydesoxybenzoin wird nun in möglichst wenig warmem 95 prozentigen Alkohol gelöst, filtriert und möglichst langsam erkalten gelassen.

Dabei fällt das Monojod-p-oxydesoxybenzoin in rosa gefärbten, glänzenden Nadeln aus, die sich meist zu größeren Kristallaggregaten vereinigen.

Diese Nadeln schmelzen bei $195^{\circ}$ (korr.).

Das Monojod-p-oxydesoxybenzoin ist in warmem Alkohol, in Eisessig und Äther leicht löslich, sehr schwer in siedendem Wasser, gar nicht in kaltem. Die Ausbeute betrug 90\% der Theorie.

Die Analyse der bei $100^{\circ}$ getrockneten Substanz ergab nachfolgende Werte:

I. $0 \cdot 3042 g$ Substanz gaben $0 \cdot 2116 g$ Jodsilber.

II. $0 \cdot 2987 \mathrm{~g}$ Substanz gaben $0 \cdot 2080 \mathrm{~g}$ Jodsilber.

In 100 Teilen:

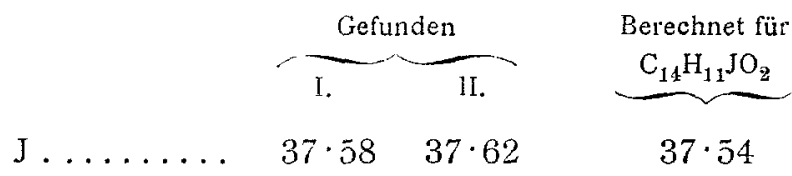

Berechnet für 
Auch hier war das Jod in die Methylengruppe eingetreten, da die alkoholische Lösung mit Silbernitrat eine quantitative Abscheidung von Jodsilber ergab.

$0.3055 \mathrm{~g}$ der bei $100^{\circ}$ getrockneten Substanz gaben $0.2131 \mathrm{~g}$ Jodsilber.

In 100 Teilen:

J.......37.69 $\underbrace{\text { Gefunden }}_{37 \cdot 54}$

\section{Darstellung des Phenylessigsäurephenylesters.}

Wie schon im theoretischen Teil erwähnt wurde, habe ich den mit dem $p$-Oxydesoxybenzoin isomeren Phenylester der Phenylessigsäure durch Kondensation der Komponenten mit Phosphorpentoxyd dargestellt.

$30 \mathrm{~g}$ Phenylessigsäure und $20.5 \mathrm{~g}$ Phenol werden in $200 \mathrm{~g}$ Chloroform gelöst und bis zum Siedepunkte des Chloroforms auf dem Wasserbade erwärmt.

Sodann trägt man die fünffache theoretische Menge von Phosphorpentoxyd (117g) in sehr kleinen Portionen ein.

Die erzeugte Reaktionswärme genügt zur Vollendung des Prozesses und ist weiteres Erwärmen nicht nötig. Das einfallende Phosphorpentoxyd bewirkt unter Zischen die Kondensation, und es scheidet sich eine hochrot gefärbte feste Verbindung aus.

Man kommt mit der theoretischen Menge von Phosphorpentoxyd deshalb nicht aus, weil die entstehende Verbindung mechanisch unangegriffenes Phosphorpentoxyd einschließt. Aus diesem Grunde empfiehit sich auch häufiges Umschütteln.

Ist alles Phosphorpentoxyd eingetragen, so wird das feste Reaktionsprodukt im selben Kolben noch durch Zugabe von Wasser zerlegt, nachdem man die Hauptmenge des Chloroforms abgegossen hat.

Der Phenylessigsäurephenylester scheidet sich als gelbes, schweres Öl ab, das nun in dem früher abgegossenen Chloroform gelöst wird und in einem Scheidetrichter wiederholt mit 
verdünnter Kalilauge $(D=1 \cdot 07)$ gewaschen wird, um die gebildete Phosphorsäure, eventuell auch unangegriffene Ausgangsmaterialien $\mathrm{zu}$ entfernen.

Durch Waschen mit Wasser verdrängt man die Kalilauge, trocknet dann die Chloroformlösung über Calciumchlorid und unterwirft sie der fraktionierten Destillation bei gewöhnlichem Druck.

Zunächst geht, auf dem Wasserbad erwärmt, das Chloroform über, dann erhitzt man mit freier Flamme, wobei die Flüssigkeit erst bei $260^{\circ}$ ins Sieden gerät.

$Z w$ ischen $260^{\circ}$ bis $320^{\circ}$ geht ein hellgelbes, schweres Öl über. Bei $325^{\circ}$ tritt Bräunung und bald darauf Verkohlung ein.

Das überdestillierte Öl erstarrt nach kurzer Zeit zu gelb gefärbten Nadeln, die abgesaugt, von der Mutterlauge befreit, in Äther gelöst und mit verdünnter Kalilauge gewaschen werden.

Man verdrängt die Kalilauge durch Wasser, läßt die ätherische Schicht $a b$ und überläßt sie der freiwilligen Verdunstung.

Alsbald scheiden sich drusenförmige, rein weiße Kristallgruppen des Phenylessigsäurephenylesters ab, die über Schwefelsäure getrocknet, bei $35^{\circ}$ (korr.) schmelzen.

Der Phenylester der Phenylessigsäure ist in Wasser, Alkalien und verdünnten Säuren unlöslich, schwer löslich in kaltem Äther, leicht in Alkohol, Eisessig und Petroläther.

Die Ausbeute betrug $85 \%$ der Theorie.

Die Analyse der über Schwefelsäure getrockneten Substanz ergab nachfolgende Werte:

I. $0.2004 \mathrm{~g}$ Substanz gaben $0.5826 \mathrm{~g}$ Kohlensäure und $0.0981 \mathrm{~g}$ Wasser

II. $0 \cdot 2005 g$ Substanz gaben $0.5827 g$ Kohlensäure und $0.0994 \mathrm{~g}$ Wasser.

In 100 Teilen:

\begin{tabular}{|c|c|c|}
\hline & Gefunden & Berechnet für \\
\hline & II & $\mathrm{C}_{14} \mathrm{H}_{12} \mathrm{O}_{2}$ \\
\hline$C$. & $79 \cdot 30 \quad 79 \cdot 26$ & $79 \cdot 24$ \\
\hline$\ldots$ & $5 \cdot 44 \quad 5 \cdot 52$ & $5 \cdot 66$ \\
\hline
\end{tabular}




\section{Spaltung des Phenylessigsäurephenylesters.}

$1 g$ Phenylessigsäurephenylester wird mit $10 \mathrm{~cm}^{3}$ wässeriger Kalilauge $(D=1 \cdot 2)$ am Rückflußkühler so lange zum Sieden erhitzt, bis kein Öltröpfchen des Esters mehr sichtbar ist. Dies ist ungefähr in einer halben Stunde der Fall.

Man verdünnt nun etwas mit Wasser, säuert mit verdünnter Schwefelsäure an und versetzt sodann bis zur alkalischen Reaktion mit Natriumcarbonatlösung $(D=1 \cdot 1)$, wodurch das Natriumsalz der Phenylessigsäure entsteht, während Phenol nun in Freiheit bleibt.

Äthert man aus, so geht nur das Phenol in Lösung.

Die ätherische Schicht wird nach dem Waschen mit Wasser verdunstet. Phenol bleibt zurück und kann leicht als Tribromphenol mit Bromwasser nachgewiesen werden.

Die vorher vom Äther abgelassene Natriumcarbonatlösung wird nun wieder angesäuert, und die Phenylessigsäure in Freiheit gesetzt.

Man engt die Lösung auf dem Wasserbade ein; beim Erkalten fällt die Phenylessigsäure aus; sie wird einmal aus Wasser umkristallisiert und gibt dann den richtigen Schmelzpunkt von $76 \cdot 5^{\circ}$ (korr.).

Weitere Mitteilungen aus diesem Gebiete bleiben vorbehalten.

Zum Schlusse sei mir noch gestattet, meinem verehrten Lehrer Herrn Prof. Dr. W. Suida für seine Anregung und Ratschläge meinen, verbindlichsten Dank auszusprechen. 